Marquette University

e-Publications@Marquette

School of Dentistry Faculty Research and

Publications

Dentistry, School of

$4-2020$

\title{
Critical-Sized Bone Defects Regeneration Using a Bone-Inspired 3D Bilayer Collagen Membrane in Combination with Leukocyte and Platelet-Rich Fibrin Membrane (L-PRF): An In Vivo Study
}

\author{
Farahnaz Fahimipour \\ Marquette University \\ Farshid Bastami \\ Shahid Beheshti University \\ Ahad Khoshzaban \\ Tehran University of Medical Sciences \\ Shahrbanoo Jahangir \\ Iran University of Medical Sciences \\ Mohamadreza Baghaban Eslaminejad \\ Royan Institute for Stem Cell Biology and Technology, Tehran
}

See next page for additional authors

Follow this and additional works at: https://epublications.marquette.edu/dentistry_fac

Part of the Dentistry Commons

\section{Recommended Citation}

Fahimipour, Farahnaz; Bastami, Farshid; Khoshzaban, Ahad; Jahangir, Shahrbanoo; Eslaminejad, Mohamadreza Baghaban; Kahyyatan, Fahimeh; Safiaghdam, Hannaneh; Sadooghi, Yeganeh; Safa, Majid; Kashi, Tahereh S. Jafarzadeh; Dashtimoghadam, Erfan; and Tayebi, Lobat, "Critical-Sized Bone Defects Regeneration Using a Bone-Inspired 3D Bilayer Collagen Membrane in Combination with Leukocyte and Platelet-Rich Fibrin Membrane (L-PRF): An In Vivo Study" (2020). School of Dentistry Faculty Research and Publications. 444.

https://epublications.marquette.edu/dentistry_fac/444 


\section{Authors}

Farahnaz Fahimipour, Farshid Bastami, Ahad Khoshzaban, Shahrbanoo Jahangir, Mohamadreza Baghaban Eslaminejad, Fahimeh Kahyyatan, Hannaneh Safiaghdam, Yeganeh Sadooghi, Majid Safa, Tahereh S. Jafarzadeh Kashi, Erfan Dashtimoghadam, and Lobat Tayebi 
Marquette University

e-Publications@Marquette

\section{Dentistry Faculty Research and Publications/School of Dentistry}

This paper is NOT THE PUBLISHED VERSION.

Access the published version via the link in the citation below.

Tissue and Cell, Vol. 63 (April 2020): 101326. DOI. This article is C Elsevier and permission has been granted for this version to appear in e-Publications@Marquette. Elsevier does not grant permission for this article to be further copied/distributed or hosted elsewhere without express permission from Elsevier.

\section{Critical-Sized Bone Defects Regeneration Using a Bone-Inspired 3D Bilayer Collagen Membrane in Combination with Leukocyte and Platelet-Rich Fibrin Membrane (L-PRF): An In Vivo Study}

Farahnaz Fahimipour

Dental Biomaterials Department, School of Dentistry, Tehran University of Medical Sciences, Tehran, Iran

Marquette University School of Dentistry, Milwaukee, WI

Department of Chemistry, University of North Carolina at Chapel Hill, NC

Farshid Bastami

Dental Research Center, Research Institute of Dental Sciences, School of Dentistry, Shahid Beheshti University of Medical Sciences, Tehran, Iran

Oral and Maxillofacial Surgery Department, School of Dentistry, Shahid Beheshti University of Medical Sciences, Tehran, Iran

Ahad Khoshzaban

Dental Biomaterials Department, School of Dentistry, Tehran University of Medical Sciences, Tehran, Iran 
Iranian Tissue Bank and Research Center, Imam Khomeini Medical Complex Hospital, Tehran University of Medical Sciences, Tehran, Iran

Arcazistsazeh Research Center\& Industry complex, Tehran, Iran

Shahrbanoo Jahangir

Department of Tissue Engineering and Regenerative Medicine, Faculty of Advanced Technologies in Medicine, Iran University of Medical Sciences, Tehran, Iran

Department of Stem Cells and Developmental Biology, Cell Science Research Center, Royan Institute for Stem Cell Biology and Technology, ACECR, Tehran, Iran

Mohamadreza Baghaban Eslaminejad

Department of Stem Cells and Developmental Biology, Cell Science Research Center, Royan Institute for Stem Cell Biology and Technology, ACECR, Tehran, Iran

Fahimeh Khayyatan

Department of Stem Cells and Developmental Biology, Cell Science Research Center, Royan Institute for Stem Cell Biology and Technology, ACECR, Tehran, Iran

Hannaneh Safiaghdam

Students Research Committee, School of Dentistry, Shahid Beheshti University of Medical Sciences, Tehran, Iran

Yeganeh Sadooghi

Dental Biomaterials Department, School of Dentistry, Tehran University of Medical Sciences, Tehran, Iran

Majid Safa

Department of Tissue Engineering and Regenerative Medicine, Faculty of Advanced Technologies in Medicine, Iran University of Medical Sciences, Tehran, Iran

Department of Hematology and Blood Banking, Faculty of Allied Medicine, Iran University of Medical Sciences, Tehran, Iran

Tahereh S. Jafarzadeh Kashi

Dental Biomaterials Department, School of Dentistry, Tehran University of Medical Sciences, Tehran, Iran

Iranian Tissue Bank and Research Center, Imam Khomeini Medical Complex Hospital, Tehran University of Medical Sciences, Tehran, Iran

Erfan Dashtimoghadam

Marquette University School of Dentistry, Milwaukee, WI

Department of Chemistry, University of North Carolina at Chapel Hill, NC

Lobat Tayebi

Marquette University School of Dentistry, Milwaukee, WI

\begin{abstract}
Objectives

We aim to develop a 3D-bilayer collagen (COL) membrane reinforced with nano beta-tricalciumphosphate ( $\mathrm{n} \beta$-TCP) particles and to evaluate its bone regeneration in combination with leukocyteplatelet-rich fibrin (L-PRF) in vivo.
\end{abstract}




\section{Background data}

L-PRF has exhibited promising results as a cell carrier in bone regeneration in a number of clinical studies, however there are some studies that did not confirm the positive results of L-PRF application.

\section{Methods}

Mechanical \& physiochemical characteristics of the COL/n $\beta$-TCP membrane $(1 / 2 \& 1 / 4)$ were tested. Proliferation and osteogenic differentiation of seeded cells on bilayer collagen/n $\beta$-TCP thick membrane was examined. Then, critical-sized calvarial defects in 8 white New Zealand rabbits were filled with either Col, Col/n $\beta$-TCP, Col/n $\beta$-TCP combined with L-PRF membrane, or left empty. New bone formation (NBF) was measured histomorphometrically $4 \& 8$ weeks postoperatively.

\section{Results}

Compressive modulus increases while porosity decreases with higher $\beta$-TCP concentrations. Mechanical properties improve, with $89 \%$ porosity (pore size $\sim 100 \mu \mathrm{m}$ ) in the bilayer-collagen/n $\beta$-TCP membrane. The bilayer design also enhances the proliferation and ALP activity. In vivo study shows no significant difference among test groups at 4 weeks, but Col/n $\beta-T C P+L-P R F$ demonstrates more NBF compared to others $(P<0.05)$ after 8 weeks.

\section{Conclusion}

The bilayer-collagen/n $\beta$-TCP thick membrane shows promising physiochemical in vitro results and significant NBF, as $3 / 4$ of the defect is filled with lamellar bone when combined with L-PRF membrane.

\section{Keywords}

Bone regeneration, Leukocyte and platelet-rich fibrin, Beta-Tricalcium phosphate, Rabbit

\section{Introduction}

In the triad of bone tissue engineering-stem cells, growth factors and scaffold - the latter has become the interest of researchers in recent years (Bastami et al., 2017). Scaffolds harbor stem cells and promote their differentiation and proliferation, allow vascularization and de novo bone formation, may be used for local delivery of growth factors and, finally, endure mechanical forces until they degrade (Fahimipour et al., 2017). Various scaffold designs and chemical compositions have been introduced, and their physical and biological characteristics have been evaluated in vitro, in vivo and clinically in the maxillofacial area (Pang et al., 2013; Yu et al., 2014); (Wang et al., 2014). A three-dimensional (3D) scaffold must be biocompatible and osteo-inductive. It is also favorable that the scaffold degrades at the same pace as new bone is formed. Its mechanical properties should also render the scaffold strong enough to bear stresses, while its porosity allows vessel infiltration and decent nutrient distribution (Arahira and Todo, 2014). Many studies have shown bone regeneration in critical-sized defects using different scaffold templates, but there are still shortcomings regarding mentioned qualifications (Rai et al., 2007; Zhang et al., 2016).

in vivo tissue engineering studies in the realm of oral and maxillofacial reconstruction have illustrated successful applications of different biomaterials, like natural and synthetic polymers, ceramics or composites (Dey et al., 2018; Akhlaghi et al., 2019; Jazayeri et al., 2017; Dong et al., 2002). 
Beta-tricalcium phosphate ( $\beta$-TCP, $\beta-\mathrm{Ca} 3(\mathrm{PO} 4) 2)$ is among the calcium phosphates that have been used extensively in bone tissue engineering (Eftekhari et al., 2015; Chazono et al., 2004). $\beta$-TCP has demonstrated osteo-conductive capability, phase stability and firm attachment to existing bone tissue (La Monaca et al., 2018; Annibali et al., 2015; Lai et al., 2019).

$\beta$-TCP has a Ca/P ratio similar to that of natural bone tissue (Suzuki et al., 2008). Moreover, it has displayed progressive biodegradability accompanied simultaneously by formation of normal bone structure in vivo (Tanaka et al., 2017).

Collagen is the most common natural polymer used, in combination with composite scaffolds (Nikpour P et al., 2018). Many studies have shown that addition of collagen has the advantage of enhancing compressive modulus and strength of the scaffold, as well as promoting proliferation and expression of osteo-conductive factors (Hao et al., 2010; Liao et al., 2014; Zhou et al., 2007). Collagen type I is commercially available for delivery of bioactive molecules in bone tissue engineering (Friess et al., 1999). Although collagen can promote bone formation, its mechanical properties as a scaffold are less than favorable, especially for use in load-bearing areas (Bulgin et al., 2013). Thus, it has been prepared in combination with $\beta$-TCP in various ratios and through different methods (Sun et al., 2003; Xiang et al., 2003; Brkovic et al., 2008; Lee and Kim, 2011). A homogenous and well-integrated structure is desired when preparing these scaffolds, so that scaffold properties (such as the porosity and weight ratios) are reliable (Zou et al., 2005). These scaffolds are commonly fabricated as collagen coated $\beta$-TCP (Hasani-Sadrabadi et al., 2014; Deschamps et al., 2017), $\beta$-TCP coated collagen (Murakami et al., 2017; Al-Munajjed and O'Brien, 2009) or composites (Inzana et al., 2014; Du et al., 2000; Kang et al., 2015). Bilayer collagen membrane has been used in the treatment of periodontal bony defects. Collagen can readily adapt to bone surface, and new bone formation has been observed (Camelo et al., 2001; Burns et al., 2000; Sakata et al., 2006). Reinforcing the bilayer collagen design with $\beta$-TCP particles provides a construct mimicking natural bone structure.

Leukocyte platelet-rich fibrin (L-PRF) is the second generation of platelet concentrates, which were first introduced in 2001 by Choukroun et al. in the regeneration of oral and maxillofacial defects (Choukroun et al., 2001). This complex network of autologous fibrin is a favorable carrier capable of releasing growth factors within 1-4 weeks and promoting micro-vascularization, bone growth and maturation (Dohan et al., 2006). This concentrate contains cytokines that have been shown to have anti-inflammatory and osteo-inductive characteristics (Saluja et al., 2011). L-PRF has exhibited promising results as a cell carrier in bone regeneration in a number of clinical studies (Öncü and Alaaddinoglu, 2015; Pradeep et al., 2015; Kumar et al., 2015). Kim et al. demonstrated promising results in the application of L-PRF combined with TCP in rabbits (Kim et al., 2014). Similarly, in another study, L-PRF proved equally successful in regenerating rabbit calvarial defects compared to hydroxyapatite TCP scaffolds. The most desirable results, however, were obtained when the defects were filled with the combination of those (Yilmaz et al., 2014).

In this study, a 3D collagen scaffold has been fabricated and reinforced with $\beta$-TCP nano-particles. A thorough characteristic evaluation of the new design was conducted, and its osteoconductive capability was tested in vitro. Finally, critical-sized defects in rabbit calvarias were grafted by the designed thick membrane combined with L-PRF membrane to evaluate in vivo bone formation (Fig. 1). 
Bone Inspired Bilayer Collagen nano BTCP membrane

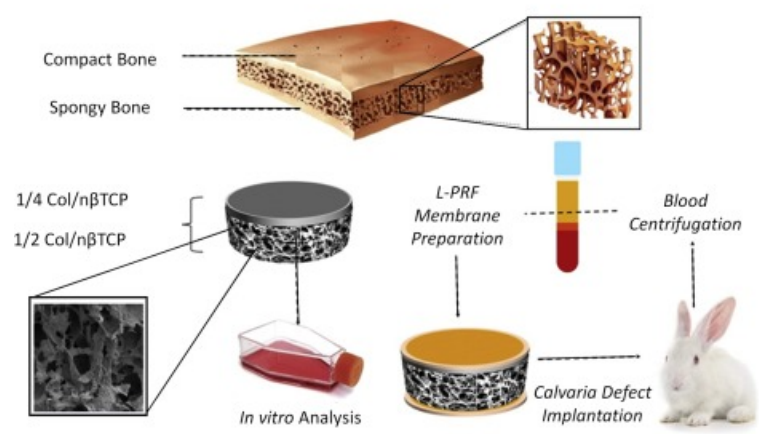

Fig. 1. Schematic design of experiment.

\section{Materials and Methods}

\subsection{Bilayer Thick Membrane Fabrication}

\subsubsection{B -TCP Collagen Bilayer Membrane}

Collagen type I solution (Sigma, USA), $50 \mathrm{mg} / \mathrm{mL}$, was dispersed in $30 \mathrm{~mL}$ of $0.1 \mathrm{M}$ acetic acid in deionized water $(\mathrm{pH}=1-4)$ at room temperature. This concentration of collagen was the maximum attainable while still maintaining an even dispersion. Mixing was performed using a homogeniser.

Calcium phosphate powders (relative ratios of 1.5 and 1.6) were synthesized by ethanol-based sol-gel method with phosphorus pentoxide $\left(\mathrm{P}_{2} \mathrm{O}_{5}, \mathrm{MERCK}\right)$ and calcium nitrate $\left(\mathrm{Ca}\left(\mathrm{NO}_{3}\right)_{2} .4 \mathrm{H}_{2} \mathrm{O}, \mathrm{MERCK}\right)$ as the chemical precursors, respectively (Öncü and Alaaddinoglu, 2015). Briefly, the $0.5 \mathrm{M}$ phosphorus pentoxide (aqueous solution) and 1.5, 1.6 and $1.67 \mathrm{M}$ of calcium nitrate in pure ethanol were obtained separately. Then phosphorus solution was slowly added into the calcium containing solution and stirred using a mechanical stirrer $(1000 \mathrm{rpm})$. The obtained sol was stirred for another $30 \mathrm{~min}$. The resulting transparent sol was kept in a closed container for $48 \mathrm{~h}$ at room temperature to obtain gel through aging process. After drying the samples in a $60^{\circ} \mathrm{C}$ oven for 1 day, the powders were kept at $120^{\circ} \mathrm{C}$ for $3 \mathrm{~h}$ to be calcined. The temperature was later increased with heating rate of $1{ }^{\circ} \mathrm{C} / \mathrm{min}$ up to $600{ }^{\circ} \mathrm{C}$ and held at this temperature for $1 \mathrm{~h}$. The final step utilized a press machine (pressure of $0.15 \mathrm{MPa}$ ), and the powders were stored at $1100^{\circ} \mathrm{C}$ for $3 \mathrm{~h}$ (Pradeep et al., 2015). $\beta$-TCP reinforcements were then added at $1 / 2$ or $1 / 4$ (collagen/ $\beta$-TCP by weight), and then the solution was mixed for another $2 \mathrm{~min}$. The collagen/ $\beta$-TCP solution was degassed in a vacuum desiccator for $15 \mathrm{~min}$. After a uniformly suspending mixture was formed, the mixture was poured into separate cylindrical silicone molds, with or without $\beta$-TCPs. The molds were placed in a freeze dryer (Labconco Inc., Kansas City, MO) on a specimen plate and cooled to $-80^{\circ} \mathrm{C}$. Scaffolds were lyophilized for $24 \mathrm{~h}$ and stored at $0{ }^{\circ} \mathrm{C}$ until further use.

In order to fabricate the bone-inspired bilayer collagen/n $\beta$-TCP membrane, a novel method with two freeze drying cycles was performed. First, the collagen/n $\beta$-TCP membrane with the ratio of $1 / 4$ was fabricated. The freeze-dried $1 / 4$ collagen/n $\beta$-TCP membrane was placed on top of the $1 / 2$ collagen $/ \mathrm{n} \beta$ TCP solution before freezing. After that, the bilayer membrane was frozen at $-80{ }^{\circ} \mathrm{C}$, lyophilized for $24 \mathrm{~h}$ and stored at $0{ }^{\circ} \mathrm{C}$ until use. All scaffolds were cross-linked using EDC/NHS in order to stiffen the collagen network. Pure collagen scaffolds were prepared as a control, and the two test groups were reinforced with $\beta$-TCP powder at $1 / 2$ and $1 / 4$ ratios (collagen/ $\beta$-TCP by weight), respectively. Scaffolds 
at $1 / 2$ and $1 / 4$ ratio approximated lower and upper bounds of the mineral content present in the ECM of natural bone (Kumar et al., 2015).

\subsection{Membrane characterization}

\subsubsection{Structure, morphology and surface topography}

Evaluation of the scaffold's morphology and surface roughness were conducted by Scanning Electron Microscopy (SEM). Three of the scaffolds were randomly selected, and the dimensions of the pore size, distance between strands and the thickness of strands in scaffolds were measured using Image J software.

\subsubsection{Porosity and mechanical properties}

Porosity was measured using solvent displacement method, as previously reported (Kim et al., 2014). Briefly, scaffolds were weighed in dry state, then immersed in $10 \mathrm{ml}$ ethanol. The specimens containing ethanol were weighed and porosity of scaffolds was calculated using the following equation:

where $W_{w}$ and $W_{d}$ are the weight of scaffold in wet and dry state, $\rho$ is the solvent density, while $R$ and $T$ are the radius and thickness of scaffolds, respectively.

The compressive modulus and strength of the collagen-based matrices and Col/n $\beta$-TCP constructs were measured utilizing a mechanical testing machine (Shimadzu, Japan) with cross-head speed of $1.0 \mathrm{~mm} \cdot \mathrm{min}^{-1}$ and a $5 \mathrm{kN}$ load cell. The cylindrical samples $(n=5)$ were compressed until they crushed.

\section{In vitro analysis}

\subsection{Cell biocompatibility}

At the first step, scaffolds were disinfected by triple immersion in $70 \%$ ethanol, followed by rinsing with PBS for 15 min cycles and air drying. $0.5 \times 106$ third passaged rabbit bone marrow derived mesenchymal stem cells (MSCs) were seeded on the scaffolds and immersed in a growth medium that was refreshed every other day. [3-(4,5-dimethylthiazol-2-yl)-1,5-diphenyltetrazulium bromide] (MTT, Sigma, USA) mitochondrial reaction method was used to determine the biocompability of the scaffold. Briefly, after $24 \mathrm{~h}$ of cellular cultivation, the scaffolds were washed with PBS and incubated with MTT solution ( $5 \mathrm{mg} / \mathrm{ml}$ in PBS) for $2 \mathrm{~h}$ at $37^{\circ} \mathrm{C}$. The MTT solution was replaced with $0.5 \mathrm{ml}$ of dimethylsulphoxide (DMSO) as an extraction solution. The absorbance of the supernatant at $540 \mathrm{~nm}$ was recorded by a spectrophotometric plate reader (Synergy HTX, BioTEK).

\subsection{Cell proliferation and cell adhesion}

PrestoBlue ${ }^{\circledR}$ (PB) assay (Invitrogen, USA) was carried out according to the manufacturer's instructions at different time intervals of 7 and 14 days after cell culture. The scaffolds were disinfected using $70 \%$ ethanol, as described above. Afterward, $1 \times 10^{6}$ cells were seeded onto the scaffolds, which were placed in a non-adherent 12 wells-plate and then submerged in culture medium. The medium was changed every other day. After the incubation period, $10 \%$ PrestoBlue was added to the scaffold-cell constructs. The fluorescence measurements (Ex: $560 \mathrm{~nm}$ and Em: $590 \mathrm{~nm}$ ) were determined by a spectrophotometric plate reader (Synergy HTX, BioTEK). In order to record the difference between study groups, triplicate samples were analyzed for this experiment. 
The attachment of cells on the scaffolds was examined by scanning electron microscopy (SEM). To do this, scaffolds were removed from the culture medium, washed in PBS and fixed with $4 \%$ glutaraldehyde. The seeded constructs were then dehydrated in gradient ethanol concentrations of 50, $70,80,90$ and $100 \%$ to conserve their intact morphology. Samples were coated with gold by means of a GSL-1100X-SPC12 Compact Plasma Sputtering Coater instrument and scanned for their microstructure using scanning electron microscope (SEM, JEOL JSM-6510LV).

\subsection{Cell differentiation}

ALP activity of cells cultured on scaffolds was assessed utilizing an ALP assay kit (Abcam, USA). The samples were incubated in osteogenic medium and tested after 7 and 14 days to evaluate osteogenic differentiation. The cell-scaffold constructs were washed with PBS, and lysis buffer solution was added to lyse the attached cells. After sonication and centrifugation, $50 \mu \mathrm{l}$ of supernatant reacted with $150 \mu \mathrm{l}$ of $p$-nitrophenyl phosphate ( $p$-NPP) for $30 \mathrm{~min}$ at $37^{\circ} \mathrm{C}$. The reaction was terminated with stop solution, and the absorbance of p-nitrophenol was measured at $405 \mathrm{~nm}$ employing a microplate reader (Synergy HTX, BioTEK) to indicate the ALP quantity. Cell lysates were analyzed for protein content using a micro-BCA Assay kit (Pierce), and ALP activity was normalized to the total protein content; alkaline phosphatase activity is expressed in arbitrary units. The results represent the mean values of three individual experiments and each in quadruplicate.

\section{In vivo analysis}

The study protocol was approved by the Ethics Committee at Tehran University of Medical Sciences. Eight New Zealand white albino rabbits were maintained and operated in accordance with the guidelines of the Institutional Animal Care and Use Committee Tehran University of Medical Sciences and the standards of the Association for Assessment and Accreditation of Laboratory Animal Care.

\subsection{L-PRF preparation}

The L-PRF was prepared via a single centrifugation of blood according to the approved protocol for a period of $12 \mathrm{~min}$ at $2700 \mathrm{rpm}$. The animal blood was taken in $9 \mathrm{~mL}$ tubes, centrifuged, and used for the experimental sites. After centrifugation, each L-PRF clot was separated from the centrifuged blood samples. Afterward, the L-PRF clot was condensed and molded on a sterile surgical plate and used as a layer of membrane.

\subsection{Surgical procedure}

Briefly, under general anesthesia induced by intramuscular injection of $35 \mathrm{mg} / \mathrm{kg}$ ketamine and $5 \mathrm{mg} / \mathrm{kg}$ xylazine, four defects with a diameter of $8 \mathrm{~mm}$ were created on the calvaria of the rabbits using a trephine bur. Defects were randomly filled with solo collagen scaffolds (Col), collagen/nano $\beta$ TCP scaffolds (Col/n $\beta$-TCP) or Col/n $\beta$-TCP scaffolds combined with L-PRF membrane (Col/n $\beta$-TCP + L-

PRF), while the 4th defect was left empty as a negative control. Connective tissue, at first, was sutured by vicril (4-0, SUPA, Iran) to prevent displacement of the grafted materials, and skin was closed by nylon (4-0, SUPA, Iran). The rabbits recovered with no complications and were sacrificed 4 or 8 weeks postoperatively. Harvested samples were fixed and maintained in formalin (10\% wt) for further analysis. 


\subsection{Histologic and histomorphometric assessments}

The samples were decalcified in nitric acid for two weeks. Longitudinal slices were cut and embedded into paraffin blocks. Then, 5-micron thick slices were sectioned and stained with hematoxylin and eosin (H\&E), to be evaluated under a light microscope (Nikon Eclipse E400, Nikon, Samida KU, Japan). Inflammation was evaluated in two ways: infiltration of acute and chronic inflammatory cells and the intensity of inflammation (score 0-3 for $<10 \%, 10-30 \%, 30-50 \%$ and $>50 \%$ inflammation, respectively). With regard to bone regeneration, type of bone (lamellar or woven) and location of new bone formation (marginal or central) were evaluated. Foreign body reaction (presence of giant cells) was also assessed. For histomorphometric analysis, the images were taken by a digital camera (Nikon Fuji HC300 Nikon) from each defect under 4X magnification of light microscope. Computer-assisted histomorphometric analysis of the new bone formation were performed using an automated image analysis software program (IHMMA, Ver. 1, SBMU, Iran). In fact, the percentage of newly formed bone and residual materials regarding the whole defect area were reported for each specimen by an experienced oral and maxillofacial pathologist blinded to the study design.

\section{Statistical analysis}

SPSS version 21 (Microsoft, IL, USA) was used for data analysis. Data is expressed as mean \pm standard deviation. One-way and two-way analysis of variance (ANOVA) was used to investigate the quantitative data. Post-hoc comparisons were performed using Turkey's HSD test. The level of significance for all tests is set at $p<0.05$.

\section{Results}

\subsection{Physicomechanical characterization}

The porosity of collagen and $1 / 2 \mathrm{Col} / \mathrm{n} \beta$-TCP scaffolds are $96.41 \pm 2.6$ and $94.45 \pm 1.7$, respectively. The mean scaffolds porosity decreases to $62.65 \pm 3.1$ with increased $\beta$-TCP fraction to $4(p<0.05)$. The mean pore size diameter is also affected by the composition of samples. The compressive modulus enhances with increased $\beta$-TCP weight fraction $(p<0.05)$. Scaffolds reinforced with $\beta$-TCP at $1 / 2$ and $1 / 4$ ratios (collagen/ $\beta$-TCP) exhibit an increased compressive modulus compared to the bare collagen $(p<0.05)$. An improved mechanical property with appropriate porosity of $89 \%$ and a pore size of $\sim 100 \mu \mathrm{m}$ is achieved in the bilayer collagen/n $\beta$-TCP membrane design. (Fig. 2) 
A
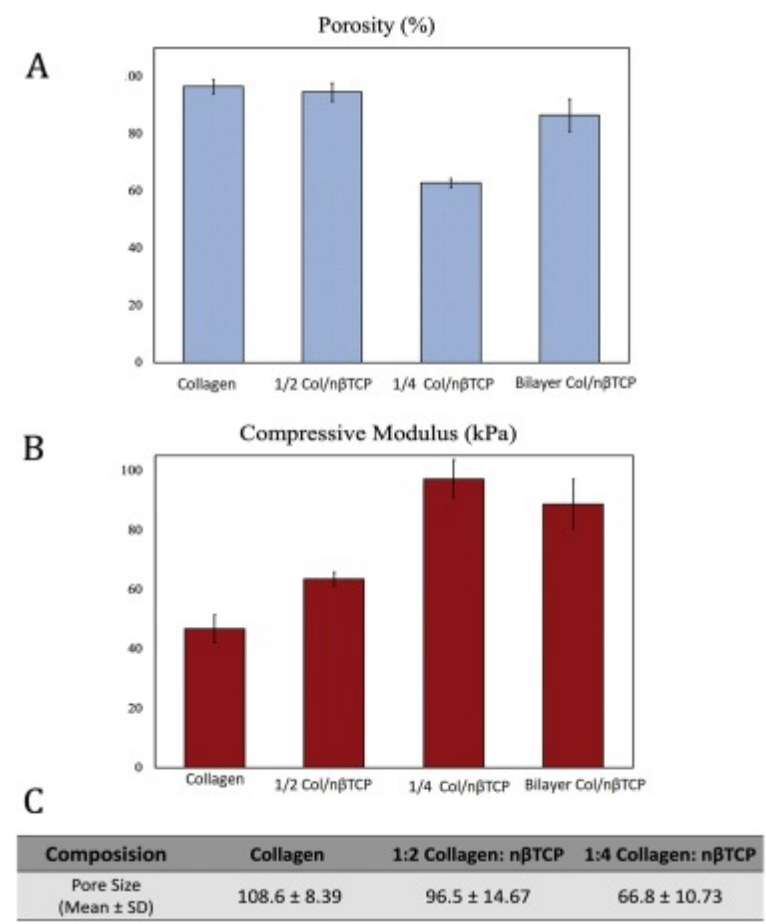

Fig. 2. (A) Porosity, (B) compressive modulus and (C) pore size of Col, Col/n $\beta-\mathrm{TCP}(1 / 2), \mathrm{Col} / \mathrm{n} \beta-\mathrm{TCP}(1 / 4)$ scaffolds.

\subsection{Biological results}

Cell biocompatibility of the scaffolds is confirmed by MTT [3-(4,5-dimethylthiazol-2-yl)-1,5-

diphenyltetrazulium bromide] assay (Fig. 3). The cellular proliferation is shown in Fig. 4A. The cells show progressive proliferation in all groups. As evident, the cells proliferate more onto the $1 / 2 \mathrm{Col} / \mathrm{n} \beta$ TCP, bilayer Col/n $\beta$-TCP than COL and $1 / 4 \mathrm{Col} / \mathrm{n} \beta$-TCP construct. The number of cells is significantly greater after 14 days compared to 7 days $(p<0.05)$. The 3D culture of the MCT3T cells on the scaffolds shows better proliferation compared to those cultured on the tissue culture plate $(p<0.05)$ (Fig. 4). Scaffold composition demonstrates a significant effect on ALP activity by MCT3T ( $p<0.05)$. By day 14 , $\beta$-TCP-containing scaffold has significantly higher ALP activity than the collagen scaffolds in osteogenic media. Cells seeded on the $1 / 4$ and bilayer thick membranes exhibit significantly higher ALP activity at both day 7 and day 14 compared to other groups ( $p<0.05$ ). By day 14 , ALP activity is also significantly higher in the $1 / 2$ group than the control ( $p<0.05$ ) (Fig. 4B). By using the bilayer design to achieve the optimal pore size and mechanical properties, the cellular proliferation and ALP activity are also optimized. 


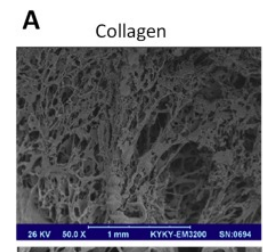

$1 / 2 \mathrm{Col} / \mathrm{n} \beta \mathrm{TCP}$

$1 / 4 \mathrm{Col} / \mathrm{n} \beta \mathrm{TCP}$
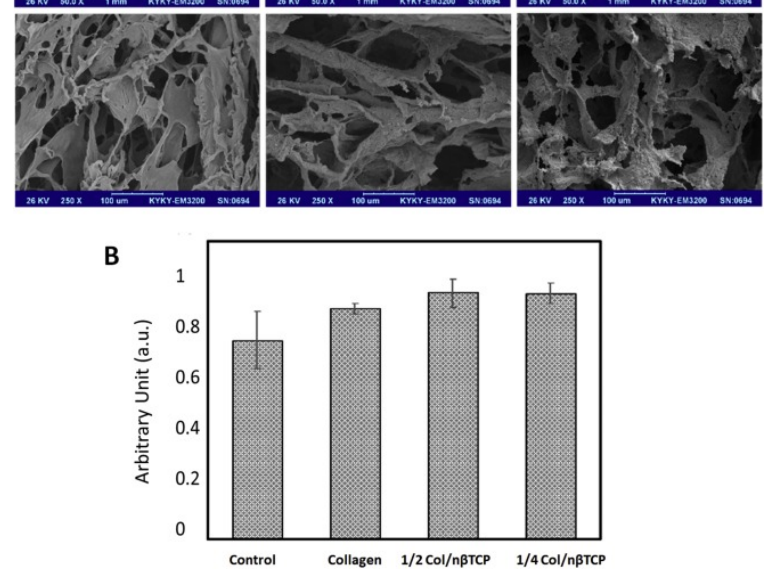

Fig. 3. (A) Scanning electron micrographs (SEM) of scaffolds with different compositions and (B) MTT assay result.
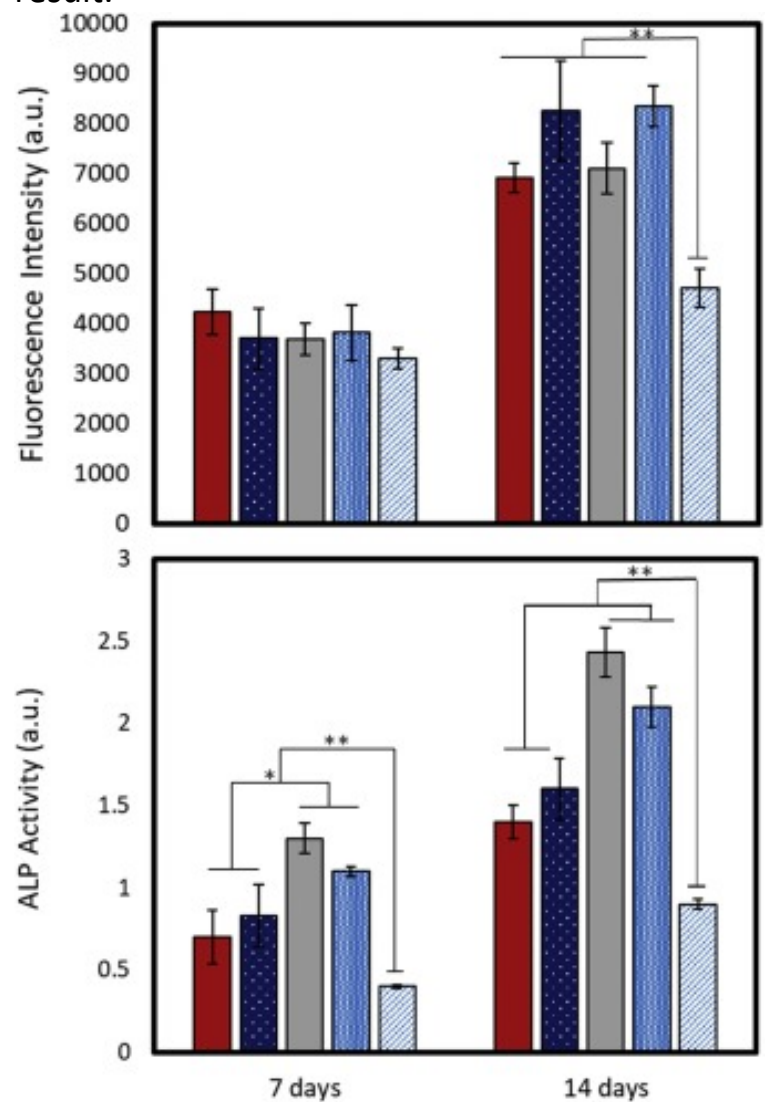

- Collagen $11 / 2 \mathrm{Col} / \mathrm{n} \beta \mathrm{TCP} \quad \square 1 / 4 \mathrm{Col} / \mathrm{n} \beta \mathrm{TCP}$

$\square$ Bilayer $\mathrm{Col} / \mathrm{n} \beta \mathrm{TCP}$ 紧 Control

Fig. 4. (A) Cellular proliferation and (B) ALP activity on the scaffolds and tissue culture dish (control). 


\subsection{In vivo results}

Minimal inflammation is observed in all study groups (below $10 \%$ ). No foreign body reaction is encountered in any of Col/n $\beta$-TCP scaffold groups. The new bone formation after 4 and 8 weeks among different groups is demonstrated in Fig. 5.
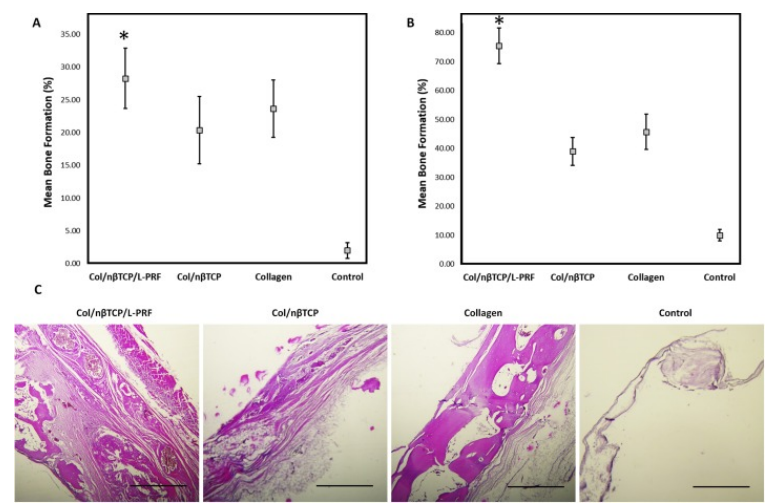

Fig. 5. New bone formation of $\mathrm{Col}, \mathrm{Col} / \mathrm{n} \beta-\mathrm{TCP}, \mathrm{Col} / \mathrm{n} \beta-\mathrm{TCP}+\mathrm{L}-\mathrm{PRF}$ and control groups after (A) 4 weeks and (B) 8 weeks, and H\&E staining after 8 weeks $(C)$.

In the Col/n $\beta$-TCP + L-PRF group, new bone formation is $28.86 \pm 2.40 \%$ and $73.36 \pm 3.74 \%$ at 4 and 8 weeks, respectively. At week 8 , newly formed bone has a fully mature lamellar structure. In the Col/n $\beta$ TCP group, new bone is measured to be $23.6 \pm 2.83 \%$ and $41.05 \pm 2.46 \%$ after 4 and 8 weeks, respectively. In Col group, $22.88 \pm 2.41 \%$ new bone is formed after 4 weeks, which is increased to $45.18 \pm 2.93 \%$ at week 8 . In the control group after 4 weeks, the defect core is empty-although a woven bony structure is developed in the peripheries, but this is statistically insignificant. At the 8th week, the percentage of newly formed bone reaches $8.7 \pm 1.29 \%$ in the peripheries, but this was of woven architecture.

\section{Discussion}

Endogenous mechanisms are found to be insufficient in healing critical-sized bony defects (Fahimipour et al., 2018, 2019), thus, various methods have been utilized to help accelerate the process.

Autogenous bone grafts are currently assumed to be the gold standard. ${ }^{20}$ However, it requires a twostep surgical procedure and has a risk of donor side morbidity and bone resorption (Shibuya and Jupiter, 2015). Given these downsides, other methods-such as xenografts and allografts-have been proposed. But slow bone formation and often unpredictable results, even rejection, is possible (Akhlaghi et al., 2019; Bauer and Muschler, 2000; Liu et al., 1997; Zioupos et al., 2008). Filling the defect with proper prefabricated scaffolds combined with the delivery of growth factors have also been proposed (Khojasteh et al., 2016). Ideally, a scaffold with suitable mechanical properties, as well as physical and biological characteristics, is desired for bone tissue engineering (Arahira and Todo, 2014).

In this study, a freeze-dried collagen scaffold seeded with $\beta$-TCP nanoparticles was fabricated. The combination of the ideal ratios of $1 / 2$ and $1 / 4$ is applied, considering the in vitro results. Later, $8 \mathrm{~mm}$ defects were created on the calvaria of New Zealand white rabbits and filled with either $\beta$ TCP/Collagen bilayer and $\beta$-TCP/Collagen + L-PRF membrane. Two remaining defects were either filled 
with solo collagen scaffold or left empty. Histomorphometric analysis was run 4 and 8 weeks postoperatively. The best results are obtained by the COL/ $\beta-T C P+L-P R F$ group. This design of the scaffold not only favors attachment and proliferation of MCT3T cells, but also shows significantly higher osteogenic ability compared to the control. Cell growth is observed after 7 days and significantly increases by day 14. MCT3T cells proliferate significantly better on the designed scaffolds as compared to culture plates.

Studies have investigated $\beta$-TCP alone or in combination with other material in shapes of granules, plates and scaffolds for bone tissue engineering (Ogose et al., 2002; Tanaka et al., 2012; Bulgin and Hodzic, 2012; Rajan et al., 2006; Tawil and Mawla, 2001). Collagen type I is a key component of natural bone extracellular matrix. Tawil et al. demonstrated that bilayer collagen membrane can help increase implant survival rate and quality of graft healing when used in sinus floor elevation (Baheiraei et al., 2018). In another study by Schwarz et al., peri-implantitis defects were treated with either nano hydroxyapatite or bovine-bone xenograft combined with bilayer collagen membrane. It was concluded that bilayer collagen membrane resulted in probing depth reduction and clinical attachment level gain after 6 months (Schwarz et al., 2006). Although collagen can promote bone formation, its low stiffness renders it mechanically weak, especially for load-bearing areas (Bulgin et al., 2013). Thus merging $\beta$ TCP nanoparticles with collagen results in a feasible scaffold with materials similar to those of natural bone and with enough strength to support different stages of bone regeneration. This bone-inspired design is our strategy in this study. A clinician must opt for a scaffold with suitable pore size to allow for vascular infiltration and bone ingrowth. In the current study, a pore size of about $60 \mu \mathrm{m}$ is observed in the specimens with $96.41(\mathrm{SD}=2.6)$ porosity, and this decreases with higher ratio of $\beta$-TCP nanoparticles.

Two different ratios of collagen to $\beta$-TCP $(1 / 2$ and $1 / 4)$ was tested in vitro to determine the most convenient. While the compressive modulus increases with higher concentrations of $\beta$-TC, density of puros decreases. The proliferation rate of cells is similar between different ratios of $\beta$-TCP scaffold, while ALP activity is significantly higher in $1 / 4$ ratio compared to other groups. Baheiraei et al. also prepared a scaffold with $1 / 4$ ratio (collagen to $\beta$-TCP particles) and concluded that addition of $\beta$-TCP to the scaffolds significantly enhanced mechanical and biological properties (Baheiraei et al., 2018;

Mojahediana et al., 2016). In our study, the compressive modulus is significantly higher in the scaffolds containing $\beta$-TCP with a mean of 83.21 (SD = 6.37) in $1 / 4$ composition compared to the collagen alone, indicating inferior mechanical characteristics of the collagen scaffolds.

Various compositions of $\mathrm{Col} / \mathrm{n} \beta$-TCP scaffold in combination with growth factors or stem cells have been documented for in vivo studies, although their fabrication process has been varied (Xiang et al., 2003; Brkovic et al., 2008; Lee and Kim, 2011; Zou et al., 2005; Fahimipour et al., 2018; Fahimipour et al., 2019; Khojasteh et al., 2017). In a study, Col/nß-TCP composite scaffolds resulted in a complete bone tissue regeneration after 12 weeks in vivo (Zou et al., 2005). In another study, rabbit tibial defects were filled with Col/n $\beta$-TCP composite scaffolds and fibroblast growth factor-2 (FGF-2). The authors observed more bone formation with addition of FGF-2 (Komaki et al., 2006). Inzana et al. used a 3D printed Col/n $\beta$-TCP scaffold and showed that the addition collagen improved flexural strength and cell viability, as well as osteo-conductivity of the scaffolds (Inzana et al., 2014). Alternatively, $\beta$-TCP particles can be coated on collagen scaffolds. Murakami et al. demonstrated that compressive 
strength, calcium ion release and enzyme resistance of $\beta$-TCP coated collagen scaffolds increased with $\beta$-TCP ratio (Murakami et al., 2017).

Another challenge that researchers encounter in regenerating bony defects is development of biological additives, such as platelet concentrate to reduce inflammation and promote healing (Miron et al., 2017). L-PRF is a fibrin matrix that acts as a reservoir of cytokines, growth factors and cells to be released in a specific period of time. This natural and economic material needs no further biomechanical modification and can be utilized as a resorbable membrane (Gassling et al., 2013). L-PRF has been used extensively in ridge augmentation procedures, sinus augmentation surgeries, guided bone regeneration (GBR) and treatment of periodontal and endodontic defects (Bastami and Khojasteh, 2016; Xuan et al., 2014). In 2012, Kim et al. used commercial TCP and L-PRF in the regeneration of bone in the calvarium defects of rabbits (Kim et al., 2012). They observed greatest new bone formation when TCP was combined with L-PRF 2, 4, 6 and 8 weeks postoperatively. Also, in our study, significantly more new bone formation is observed in Col/n $\beta-T C P+L-P R F$ groups compared to others. This newly formed bone has a lamellar structure, as opposed to the woven architecture of bone formed in the control group.

Conversely, in another study, Comert et al. found that adding PRF to $\beta$-TCP scaffolds did not significantly improve the new bone formation in maxillary sinus-floor elevation procedure (Comert Kilic et al., 2017). In another study, rabbit calvarial defects were filled with either PRF membrane or PRF and $\beta$-TCP. A third group of defects were left empty as controls. Authors argued that significant bone formation was observed in both groups compared to the control (Abdullah, 2016). In our study, minimal new bone ( $8.7 \%$ ) is formed at week 8 , which is a woven structure that is limited to peripheries.

\section{Conclusion}

Briefly, in this study, a $\beta$-TCP-reinforced collagen scaffold with desirable physical features has been fabricated. This scaffold shows promising results in cell adhesion and proliferation, as well as osteogenic differentiation. In the conducted preclinical study, up to three-fourths of the defect is regenerated with lamellar bone when the scaffold is used in combination with L-PRF membrane. The designed scaffold can be further investigated for the delivery of cells and various growth factors to initiate and advance bone tissue regeneration.

\section{Acknowledgment}

Authors have no conflict of interest to declare.

\section{References}

Abdullah, 2016. W.A. Abdullah. Evaluation of bone regenerative capacity in rats claverial bone defect using platelet rich fibrin with and without beta tri calcium phosphate bone graft material. Saudi Dent. J., 28 (2016), pp. 109-117

Akhlaghi et al., 2019. F. Akhlaghi, N. Hesami, M.R. Rad, et al. Improved bone regeneration through amniotic membrane loaded with buccal fat pad-derived MSCs as an adjuvant in maxillomandibular reconstruction. J. Cranio-maxillofacial Surg., 47 (2019), pp. 1266-1273 
Al-Munajjed and O'Brien, 2009. A.A. Al-Munajjed, F.J. O'Brien. Influence of a novel calcium-phosphate coating on the mechanical properties of highly porous collagen scaffolds for bone repair. J. Mech. Behav. Biomed. Mater., 2 (2009), pp. 138-146

Annibali et al., 2015. S. Annibali, G. Iezzi, G.L. Sfasciotti, et al. Histological and histomorphometric human results of HA-Beta-TCP 30/70 compared to three different biomaterials in maxillary sinus augmentation at 6 months: a preliminary report. Biomed Res. Int., 2015 (2015), p. 156850

Arahira and Todo, 2014. T. Arahira, M. Todo. Effects of proliferation and differentiation of mesenchymal stem cells on compressive mechanical behavior of collagen/ $\beta$-TCP composite scaffold. J. Mech. Behav. Biomed. Mater., 39 (2014), pp. 218-230

Baheiraei et al., 2018. N. Baheiraei, M.R. Nourani, S.M.J. Mortazavi, et al. Development of a bioactive porous collagen/beta-tricalcium phosphate bone graft assisting rapid vascularization for bone tissue engineering applications. J. Biomed. Mater. Res. A., 106 (2018), pp. 73-85

Bastami and Khojasteh, 2016. F. Bastami, A. Khojasteh. Use of leukocyte-and platelet-rich fibrin for bone regeneration: a systematic review. Regeneration, Reconstruction \& Restoration, 1 (2) (2016), pp. 47-68

Bastami et al., 2017. F. Bastami, Z. Paknejad, M. Jafari, et al. Fabrication of a three-dimensional $\beta$ tricalcium-phosphate/gelatin containing chitosan-based nanoparticles for sustained release of bone morphogenetic protein-2: implication for bone tissue engineering. Mater. Sci. Eng. C: C, 72 (2017), pp. 481-491

Bauer and Muschler, 2000. T.W. Bauer, G.F. Muschler. Bone graft materials. An overview of the basic science. Clin. Orthop. Relat. Res. (2000), pp. 10-27

Brkovic et al., 2008. B. Brkovic, H.S. Prasad, G. Konandreas, et al. Simple preservation of a maxillary extraction socket using beta-tricalcium phosphate with type I collagen: preliminary clinical and histomorphometric observations. J. Can. Dent. Assoc., 74 (2008), pp. 523-528

Bulgin and Hodzic, 2012. D. Bulgin, E. Hodzic. Autologous bone marrow-derived mononuclear cells combined with $\beta$-TCP for maxillary bone augmentation in implantation procedures. J. Craniofacl. Surg., 23 (2012), pp. 1728-1732

Bulgin et al., 2013. D. Bulgin, E. Irha, E. Hodzic, et al. Autologous bone marrow derived mononuclear cells combined with $\beta$-tricalcium phosphate and absorbable atelocollagen for a treatment of aneurysmal bone cyst of the humerus in child. J. Biomater. Appl., 28 (2013), pp. 343-353

Burns et al., 2000. W.T. Burns, M.E. Peacock, M.F. Cuenin, et al. Gingival recession treatment using a bilayer collagen membrane. J. Periodontol., 71 (2000), pp. 1348-1352

Camelo et al., 2001. M. Camelo, M.L. Nevins, S.E. Lynch, et al. Periodontal regeneration with an autogenous bone--Bio-Oss composite graft and a bio-gide membrane. Int. J. Periodontics Restorative Dent., 21 (2001)

Chazono et al., 2004 M. Chazono, T. Tanaka, H. Komaki, et al. Bone formation and bioresorption after implantation of injectable beta-tricalcium phosphate granules-hyaluronate complex in rabbit bone defects. J. Biomed. Mater. Res. A., 70 (2004), pp. 542-549

Choukroun et al., 2001. J. Choukroun, F. Adda, C. Schoeffler, et al. Une opportunité en paroimplantologie: le PRF. Implantodontie, 42 (2001), p. e62

Comert Kilic et al., 2017. S. Comert Kilic, M. Gungormus, S.N. Parlak. Histologic and histomorphometric assessment of sinus-floor augmentation with beta-tricalcium phosphate alone or in combination with pure-platelet-rich plasma or platelet-rich fibrin: a randomized clinical trial. Clin. Implant Dent. Relat. Res., 19 (2017), pp. 959-967 
Deschamps et al., 2017. I.S. Deschamps, G.L. Magrin, R.S. Magini, et al. On the synthesis and characterization of beta-tricalcium phosphate scaffolds coated with collagen or poly (D, Llactic acid) for alveolar bone augmentation. Eur. J. Dent., 11 (2017), pp. 496-502

Dey et al., 2018. R.E. Dey, I. Wimpenny, J.E. Gough, et al. Poly (vinylphosphonic acid-co-acrylic acid) hydrogels: the effect of copolymer composition on osteoblast adhesion and proliferation. J. Biomed. Mater. Res. A., 106 (2018), pp. 255-264

Dohan et al., 2006. D.M. Dohan, J. Choukroun, A. Diss, et al. Platelet-rich fibrin (PRF): a secondgeneration platelet concentrate. Part I: technological concepts and evolution. Oral Surg. Oral Med. Oral Pathol. Oral Radiol. Endod., 101 (2006), pp. e37-e44

Dong et al., 2002. J. Dong, T. Uemura, Y. Shirasaki, et al. Promotion of bone formation using highly pure porous beta-TCP combined with bone marrow-derived osteoprogenitor cells. Biomaterials, 23 (2002), pp. 4493-4502

Du et al., 2000. C. Du, F. Cui, W. Zhang, et al. Formation of calcium phosphate/collagen composites through mineralization of collagen matrix. J. Biomedl. Mater. Res. A, 50 (2000), pp. 518-527

Eftekhari et al., 2015. H. Eftekhari, M.R. Farahpour, S.M. Rabiee. Histopathological evaluation of potential impact of beta-tricalcium phosphate (HA+ beta-TCP) granules on healing of segmental femur bone defect. Bratisl. Med. J., 116 (2015), pp. 30-34

Fahimipour et al., 2017. F. Fahimipour, M. Rasoulianboroujeni, E. Dashtimoghadam, et al. 3D printed TCP-based scaffold incorporating VEGF-loaded PLGA microspheres for craniofacial tissue engineering. Dentl Mater, 33 (2017), pp. 1205-1216

Fahimipour et al., 2018. F. Fahimipour, E. Dashtimoghadam, M. Rasoulianboroujeni, et al. Collagenous matrix supported by a 3D-printed scaffold for osteogenic differentiation of dental pulp cells. Dent. Mater., 34 (2018), pp. 209-220

Fahimipour et al., 2019. F. Fahimipour, E. Dashtimoghadam, M.M. Hasani-Sadrabadi, et al. Enhancing cell seeding and osteogenesis of MSCs on 3D printed scaffolds through injectable BMP2 immobilized ECM-Mimetic gel. Dent. Mater., 35 (2019), pp. 990-1006

Friess et al., 1999. W. Friess, H. Uludag, S. Foskett, et al. Characterization of absorbable collagen sponges as rhBMP-2 carriers. Int. J. Pharm., 187 (1999), pp. 91-99

Gassling et al., 2013. V. Gassling, N. Purcz, J.H. Braesen, et al. Comparison of two different absorbable membranes for the coverage of lateral osteotomy sites in maxillary sinus augmentation: a preliminary study. J. Cranio-Maxillo-Fac. Surg., 41 (2013), pp. 76-82

Hao et al., 2010. W. Hao, L. Pang, M. Jiang, et al. Skeletal repair in rabbits using a novel biomimetic composite based on adipose-derived stem cells encapsulated in collagen I gel with PLGAbeta-TCP scaffold. J. Orthop. Res, 28 (2010), pp. 252-257

Hasani-Sadrabadi et al., 2014. M.M. Hasani-Sadrabadi, V. Karimkhani, F.S. Majedi, et al. Microfluidicassisted self-assembly of complex dendritic polyethylene drug delivery nanocapsules. Adv Mater., 19 (2014), pp. 3118-3123

Inzana et al., 2014. J.A. Inzana, D. Olvera, S.M. Fuller, et al. 3D printing of composite calcium phosphate and collagen scaffolds for bone regeneration. Biomaterials, 35 (2014), pp. 40264034

Jazayeri et al., 2017. H.E. Jazayeri, M. Tahriri, M. Razavi, et al. A current overview of materials and strategies for potential use in maxillofacial tissue regeneration. Mater. Sci. Eng. C, 70 (2017), pp. 913-929

Kang et al., 2015. Y. Kang, N. Mochizuki, A. Khademhosseini, et al. Engineering a vascularized collagenbeta-tricalcium phosphate graft using an electrochemical approach. Acta Biomater., 11 (2015), pp. 449-458 
Khojasteh et al., 2016. A. Khojasteh, F. Fahimipour, M.B. Eslaminejad, et al. Development of PLGAcoated $\beta$-TCP scaffolds containing VEGF for bone tissue engineering. Mater. Sci. Eng. C, 69 (2016), pp. 780-788

Khojasteh et al., 2017. A. Khojasteh, F. Fahimipour, M. Jafarian, et al. Bone engineering in dog mandible: coculturing mesenchymal stem cells with endothelial progenitor cells in a composite scaffold containing vascular endothelial growth factor. J. Biomed. Mater. Res. Part B Appl. Biomater., 105 (2017), pp. 1767-1777

Kim et al., 2012. B.J. Kim, T.K. Kwon, H.S. Baek, et al. A comparative study of the effectiveness of sinus bone grafting with recombinant human bone morphogenetic protein 2-coated tricalcium phosphate and platelet-rich fibrin-mixed tricalcium phosphate in rabbits. Oral Surg. Oral Med. Oral Pathol. Oral Radiol. Endod., 113 (2012), pp. 583-592

Kim et al., 2014. T.H. Kim, S.H. Kim, G.K. Sandor, et al. Comparison of platelet-rich plasma (PRP), platelet-rich fibrin (PRF), and concentrated growth factor (CGF) in rabbit-skull defect healing. Arch. Oral Biol., 59 (2014), pp. 550-558

Komaki et al., 2006. H. Komaki, T. Tanaka, M. Chazono, et al. Repair of segmental bone defects in rabbit tibiae using a complex of beta-tricalcium phosphate, type I collagen, and fibroblast growth factor-2. Biomaterials, 27 (2006), pp. 5118-5126

Kumar et al., 2015. N. Kumar, K. Prasad, L. Ramanujam, et al. Evaluation of treatment outcome after impacted mandibular third molar surgery with the use of autologous platelet-rich fibrin: a randomized controlled clinical study. J. Oral Maxillofac. Surg., 73 (2015), pp. 1042-1049

La Monaca et al., 2018. G. La Monaca, G. lezzi, M.P. Cristalli, et al. Comparative histological and histomorphometric results of six biomaterials used in two-stage maxillary sinus augmentation model after 6-Month healing. Biomed Res. Int., 27 (2018), pp. 943-989

Lai et al., 2019. Y. Lai, Y. Li, H. Cao, et al. Osteogenic magnesium incorporated into PLGA/TCP porous scaffold by 3D printing for repairing challenging bone defect. Biomaterials, 197 (2019), pp. 207-219

Lee and Kim, 2011. H. Lee, G. Kim. Three-dimensional plotted PCL/ $\beta$-TCP scaffolds coated with a collagen layer: preparation, physical properties and in vitro evaluation for bone tissue regeneration. J. Mater. Chem., 21 (2011), pp. 6305-6312

Liao et al., 2014. H.-T. Liao, Y.-Y. Chen, Y.-T. Lai, et al. The osteogenesis of bone marrow stem cells on mPEG-PCL-mPEG/Hydroxyapatite composite scaffold via solid freeform fabrication. Biomed Res. Int., 2014 (2014), p. 13

Liu et al., 1997. H.S. Liu, T.S. Chin, L.S. Lai, et al. Hydroxyapatite synthesized by a simplified hydrothermal method. Ceram. Int., 23 (1997), pp. 19-25

Miron et al., 2017. R.J. Miron, M. Bishara, J. Choukroun. Basics of platelet-rich fibrin therapy. Dent. Today, 36 (2017), pp. 74-76

Mojahediana et al., 2016. M. Mojahediana, F. Fahimipourb, K.L. Larsena, et al. Ethanol-based sol-gel synthesis of nano-crystalline hydroxyapatite with different calcium phosphorus ratios (Ca/P). J Ceram. Process. Res., 17 (2016), pp. 1138-1142

Murakami et al., 2017. S. Murakami, H. Miyaji, E. Nishida, et al. Dose effects of beta-tricalcium phosphate nanoparticles on biocompatibility and bone conductive ability of threedimensional collagen scaffolds. Dent. Mater. J., 36 (2017), pp. 573-583

Nikpour P et al., 2018. H. Nikpour P, H. Salimi-Kenari, F. Fahimipour, et al. Dextran hydrogels incorporated with bioactive glass-ceramic: nanocomposite scaffolds for bone tissue engineering. Carbohyd. Polym., 190 (2018), pp. 281-294 
Ogose et al., 2002. A. Ogose, T. Hotta, H. Hatano, et al. Histological examination of $\boldsymbol{\beta}$-tricalcium phosphate graft in human femur. J. Biomedl. Mater. Res. A, 63 (2002), pp. 601-604

Öncü and Alaaddinoglu, 2015. E. Öncü, E.E. Alaaddinoglu. The effect of platelet-rich fibrin on implant stability. Int J OralMaxillofac Implants, 30 (2015)

Pang et al., 2013. L. Pang, W. Hao, M. Jiang, et al. Bony defect repair in rabbit using hybrid rapid prototyping polylactic-co-glycolic acid/ $\beta$-tricalciumphosphate collagen I/apatite scaffold and bone marrow mesenchymal stem cells. Indian J. Orthop., 47 (2013), p. 388

Pradeep et al., 2015. A. Pradeep, K. Nagpal, S. Karvekar, et al. Platelet-rich fibrin with $1 \%$ metformin for the treatment of intrabony defects in chronic periodontitis: a randomized controlled clinical trial. J. Periodontol., 86 (2015), pp. 729-737

Rai et al., 2007. B. Rai, K.H. Ho, Y. Lei, et al. Polycaprolactone-20\% tricalcium phosphate scaffolds in combination with platelet-rich plasma for the treatment of critical-sized defects of the mandible: a pilot study. J. Oral Maxillofac. Surg., 65 (2007), pp. 2195-2205

Rajan et al., 2006. N. Rajan, J. Habermehl, M.-F. Coté, et al. Preparation of ready-to-use, storable and reconstituted type I collagen from rat tail tendon for tissue engineering applications. Nat. Protoc., 1 (2006), p. 2753

Sakata et al., 2006. J. Sakata, H. Abe, A. Ohazama, et al. Effects of combined treatment with porous bovine inorganic bone grafts and bilayer porcine collagen membrane on refractory one-wall intrabony defects. Int. J. Periodontics Restorative Dent., 26 (2006)

Saluja et al., 2011. H. Saluja, V. Dehane, U. Mahindra. Platelet-Rich fibrin: a second generation platelet concentrate and a new friend of oral and maxillofacial surgeons. Ann. Maxillofac. Surg., 1 (2011), p. 53

Schwarz et al., 2006. F. Schwarz, K. Bieling, T. Latz, et al. Healing of intrabony peri-implantitis defects following application of a nanocrystalline hydroxyapatite $\left(\right.$ Ostim $^{\mathrm{TM}}$ ) or a bovine-derived xenograft (Bio-Oss ${ }^{\mathrm{TM}}$ ) in combination with a collagen membrane (Bio-Gide ${ }^{\mathrm{TM}}$ ). A case series. J. Clin. Periodontol., 33 (2006), pp. 491-499

Shibuya and Jupiter, 2015. N. Shibuya, D.C. Jupiter. Bone graft substitute: allograft and xenograft. Clin. Podiatr. Med. Surg., 32 (2015), pp. 21-34

Sun et al., 2003. J.S. Sun, F.H. Lin, Y.J. Wang, et al. Collagen-hydroxyapatite/Tricalcium phosphate microspheres as a delivery system for recombinant human transforming growth Factor- $\beta$ 1. J. Artif. Organs, 27 (2003), pp. 605-612

Suzuki et al., 2008. O. Suzuki, H. Imaizumi, S. Kamakura, et al. Bone regeneration by synthetic octacalcium phosphate and its role in biological mineralization. Curr. Med. Chem., 15 (2008), pp. 305-313

Tanaka et al., 2012. T. Tanaka, S. Kitasato, M. Chazono, et al. Use of an Injectable Complex of $\boldsymbol{\beta}$ Tricalcium Phosphate Granules, Hyaluronate, and Fibroblast Growth Factor-2 on Repair of Unstable Intertrochanteric Fractures. Open Biomedl. Eng. J., 6 (2012), p. 98

Tanaka et al., 2017. T. Tanaka, H. Komaki, M. Chazono, et al. Basic research and clinical application of beta-tricalcium phosphate (beta-TCP). Morphologie, 101 (2017), pp. 164-172

Tawil and Mawla, 2001. G. Tawil, M. Mawla. Sinus floor elevation using a bovine bone mineral (BioOss) with or without the concomitant use of a bilayered collagen barrier (Bio-Gide): a clinical report of immediate and delayed implant placement. Int. J. OralMaxillofac Implants, 16 (2001)

Wang et al., 2014. J. Wang, P. Qiao, L. Dong, et al. Microencapsulated rBMMSCs/calcium phosphate cement for bone formation in vivo. Biomed. Mater. Eng., 24 (2014), pp. 835-843 
Xiang et al., 2003. L. Xiang, C. Weimin, L. Shenghong, et al. Expression of TGF- $\beta$ in region of bone defect repaired by collagen nano-beta-tricalcium phosphate composite artificial bone. J. Huazhong Univ. Sci. Technol. Med. Sci., 23 (2003), pp. 302-305

Xuan et al., 2014. F. Xuan, C.U. Lee, J.S. Son, et al. A comparative study of the regenerative effect of sinus bone grafting with platelet-rich fibrin-mixed Bio-Oss(R) and commercial fibrin-mixed Bio-Oss(R): an experimental study. J. Cranio-Maxillo-Fac. Surg., 42 (2014), pp. e47-50

Yilmaz et al., 2014. D. Yilmaz, N. Dogan, A. Ozkan, et al. Effect of platelet rich fibrin and beta tricalcium phosphate on bone healing. A histological study in pigs. Acta bras cir, 29 (2014), pp. 59-65

Yu et al., 2014. B.-H. Yu, Q. Zhou, Z.-L. Wang. Periodontal ligament versus bone marrow mesenchymal stem cells in combination with Bio-Oss scaffolds for ectopic and in situ bone formation: a comparative study in the rat. J biomater applications, 29 (2014), pp. 243-253

Zhang et al., 2016. X. Zhang, Y. Li, Y.E. Chen, et al. Cell-free 3D scaffold with two-stage delivery of miRNA-26a to regenerate critical-sized bone defects. Nat. Commun., 7 (2016), p. 10376

Zhou et al., 2007. Y. Zhou, D.W. Hutmacher, S.-L. Varawan, et al. In vitro bone engineering based on polycaprolactone and polycaprolactone-tricalcium phosphate composites. Polym. Int., 56 (2007), pp. 333-342

Zioupos et al., 2008. P. Zioupos, R.B. Cook, J.R. Hutchinson. Some basic relationships between density values in cancellous and cortical bone. J. Biomech., 41 (2008), pp. 1961-1968

Zou et al., 2005. C. Zou, W. Weng, X. Deng, et al. Preparation and characterization of porous betatricalcium phosphate/collagen composites with an integrated structure. Biomaterials, 26 (2005), pp. 5276-5284 